\title{
Trend analysis of extreme precipitation in the Northwestern Highlands of Ethiopia with a case study of Debre Markos
}

\author{
H. Shang ${ }^{1}$, J. Yan ${ }^{1,2}$, M. Gebremichael ${ }^{3}$, and S. M. Ayalew ${ }^{4}$ \\ ${ }^{1}$ Department of Statistics, University of Connecticut, Storrs, Connecticut, USA \\ ${ }^{2}$ Center for Environmental Sciences and Engineering, University of Connecticut, Storrs, Connecticut, USA \\ ${ }^{3}$ Department of Civil and Environmental Engineering, University of Connecticut, Storrs, Connecticut, USA \\ ${ }^{4}$ Department of Civil Engineering, Faculty of Technology, Addis Ababa University, Ethiopia
}

Received: 21 September 2010 - Published in Hydrol. Earth Syst. Sci. Discuss.: 28 October 2010

Revised: 28 March 2011 - Accepted: 13 June 2011 - Published: 24 June 2011

\begin{abstract}
Understanding extreme precipitation is very important for Ethiopia, which is heavily dependent on lowproductivity rainfed agriculture but lacks structural and nonstructural water regulating and storage mechanisms. There has been an increasing concern about whether there is an increasing trend in extreme precipitation as the climate changes. Existing analysis of this region has been descriptive, without taking advantage of the advances in extreme value modeling. After reviewing the statistical methodology on extremes, this paper presents an analysis based on the generalized extreme value modeling with daily time series of precipitation records at Debre Markos in the Northwestern Highlands of Ethiopia. We found no strong evidence to reject the null hypothesis that there is no increasing trend in extreme precipitation at this location.
\end{abstract}

\section{Introduction}

In Ethiopia, rainfall is by far the most important factor climate, as is true for most of Africa. Low-productivity agriculture, which accounts for a majority of the national economy, relies heavily on rainfall. Climate extremes such as drought or flood often lead to famine and disaster for the vulnerable agricultural, social and economic environment in Ethiopia, which lacks structural and non-structural water regulating

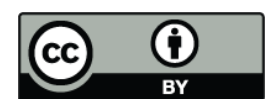

Correspondence to: J. Yan

(jun.yan@uconn.edu) and storage mechanisms. In particular, flood, as a result of extreme precipitation, poses serious threat on food security and public safety. Estimating the probability of extreme precipitation and characterizing the uncertainty of the estimates are crucial to, for instance, structural design, public safety alerts, evacuation management, and loss mitigation.

Given the increasing public concern on climate change, it is of particular interest to test whether there is a long term increasing trend in extreme precipitation. Studies have been done for different parts of the world. For examples, Kunkel et al. (1999) reported an increasing trend in the United States at a rate of $3 \%$ per decade from 1931 to 1996, but no significant trend during 1951-1993 in Canada; Kunkel (2003) showed a sizeable increase in the frequency of extreme precipitation events since the 1920s/1930s in the US; Frei and Schär (2001) found an increase in the frequency of heavy precipitation during 1901-1994 in the Alpine region of Switzerland; Goswami et al. (2006) detected a significant rising trend in both the frequency and the magnitude of extreme rainfall events from 1951 to 2000 in central India; Karagiannidis et al. (2009) reported no significant trend in extreme precipitation of the European continent from the mid 1970's to 2000 .

Both descriptive approaches and model-based approaches have been used to detect the trend in extreme precipitation. Kunkel et al. (1999) defined extreme precipitation events given a certain duration and a site-specific threshold, and tested linear trend in the frequency of extreme precipitation events using the nonparametric Mann-Kendall test. Frei and Schär (2001) used a logistic regression model to test the

Published by Copernicus Publications on behalf of the European Geosciences Union. 
long-term trend in the counts of heavy precipitation events based on a binomial model for the counts. Zhang et al. (2004) compared three methods for trend detection in extreme values in a Monte Carlo study, ordinary least squares regression, nonparametric Mann-Kendall test, and generalized extreme value (GEV) modeling. The GEV method can use the $m$ largest observations each year. Explicit GEV modeling was found to always outperform the other two methods, and the use of $m$-largest observations was found to improve the detection power for moderate values of $m$.

Rainfall patterns in Ethiopia have been reported in previous studies. A decline of annual and summer rainfall in eastern, southern, and southwestern Ethiopia was found, but no trend was detected over central, northern, and northwestern Ethiopia (Seleshi and Zanke, 2004; Cheung et al., 2008). It is worth noting, however, that annual or summer total rainfall and annual maximum daily rainfall are very different aspects of rainfall characterization. Seleshi and Camberlin (2006) studied changes in extreme seasonal rainfall as measured by extreme rainfall indices with daily rainfall data. One of the indices was extreme intensity, defined as the average intensity of events greater than or equal to the 95 th percentile. A weak increasing trend in summer extreme intensity over the $10-11^{\circ}$ North band of the Ethiopia Highlands and no trend was found over the remaining Highlands, based on the nonparametric Mann-Kendall test for trend. These existing analyses have been descriptive, without taking advantage of the advances in extreme value modeling from the statistics literature. To the best of our knowledge, extreme value analysis based on the GEV modeling has not been applied to extreme precipitation data in Ethiopia.

The GEV distribution was first introduced by Fisher and Tippett (1928) as limits of the sample maximum or minimum for independent, identically distributed variables. Extreme value theory has evolved into a proliferating field in statistics, motivated by numerous environmental applications. Accessible statistical references are, for instances, Coles (2001) and Beirlant et al. (2004). Extreme precipitation has been an important application area of extreme value analysis (e.g., Durman et al., 2001; Kharin and Zwiers, 2005; Huerta and Sansó, 2007). In particular, statistical inferences for univariate extreme value analysis, as is the case with the precipitation data at a single location, have been rather mature and widely applied by practitioners in many fields. Two standard approaches can be used to fit a univariate GEV distribution. The first one, known as the block maxima approach, applies to annual maxima of a time series, using only one data point, the maximum, per year. The second one applies to all exceedances over a high threshold, also known as "peaks over threshold" (POT). The method we adopted in this article is a variant of the POT approach, the point process approach; see Sect. 3 for more details. Compared to the $m$-largest observation approach, which can be wasteful if one block happens to contain more extreme events than another, the point process approach utilizes more information from the data. Given the relatively short period of data record, the point process approach is adopted in this application as it takes full advantage of daily precipitation record in fitting GEV distributions.

Through GEV models, this article aims to provide an extreme value analysis of the annual maximum precipitation in Debre Markos, Ethiopia. Specifically, our objective is to test whether there is an increasing trend in extreme precipitation in this area given the public concerns of suspected trend as a consequence of global climate changes. We incorporated a linear function of time in the location parameter of a GEV distribution and fitted the model with the POT approach to the daily precipitation data at Debre Markos. No evidence was found to support an increasing trend in extreme precipitation since 1953 at this location.

The rest of the article is organized as follows. Details of the data are described in Sect. 2 . The statistical methods to be used, including the extreme value theory and modeling techniques, are reviewed in Sect. 3. The results of the extreme value analysis are reported in Sect. 4 with a test for trend. A discussion concludes in Sect. 5 .

\section{Data}

Debre Markos is a city in the Blue Nile River basin on the Northwestern Highlands of Ethiopia. It has latitude $10^{\circ} 20^{\prime} \mathrm{N}$, longitude $37^{\circ} 43^{\prime} \mathrm{E}$, and elevation $2446 \mathrm{~m}$. Although the topography of Ethiopia is highly diverse, more than $45 \%$ of the country is dominated by highlands with elevations greater than $1500 \mathrm{~m}$, where almost $90 \%$ of the nation's population resides. The rain gauge station at Debre Markos provides the longest record among all stations in Ethiopia. Daily precipitation records are available from 1953, with only a tiny proportion of missing data. We use Debre Markos as a case study to investigate the long term trend in extreme precipitation in the Northwestern highland of Ethiopia.

Our raw data of daily precipitation at Debre Markos spans from 1 November 1953 to 10 December 2006. Out of the total of 19398 days, 229 (about 1.2\%) observations are missing. The observed daily time series of precipitation is plotted in Fig. 1. The maximum daily was $86.9 \mathrm{~mm}$, observed on 14 August 1997.

The daily precipitation series are obviously not independent and not identically distributed. Larger precipitations may tend to occur in clusters. For instance, out of 76 days in Junes with precipitation exceeding the 95th percentile of June precipitation, there were 9 occasions of two or more consecutive exceedances. These counts are 7 out of 79, 3 out of 78 , and 7 out of 80 for July, August, and September, respectively, the other three most rainy months. If there were no temporal dependence, $5 \%$ percent of the exceedences would be expected to be followed by another exceedance. The relative frequencies of clustered exceedances are higher 


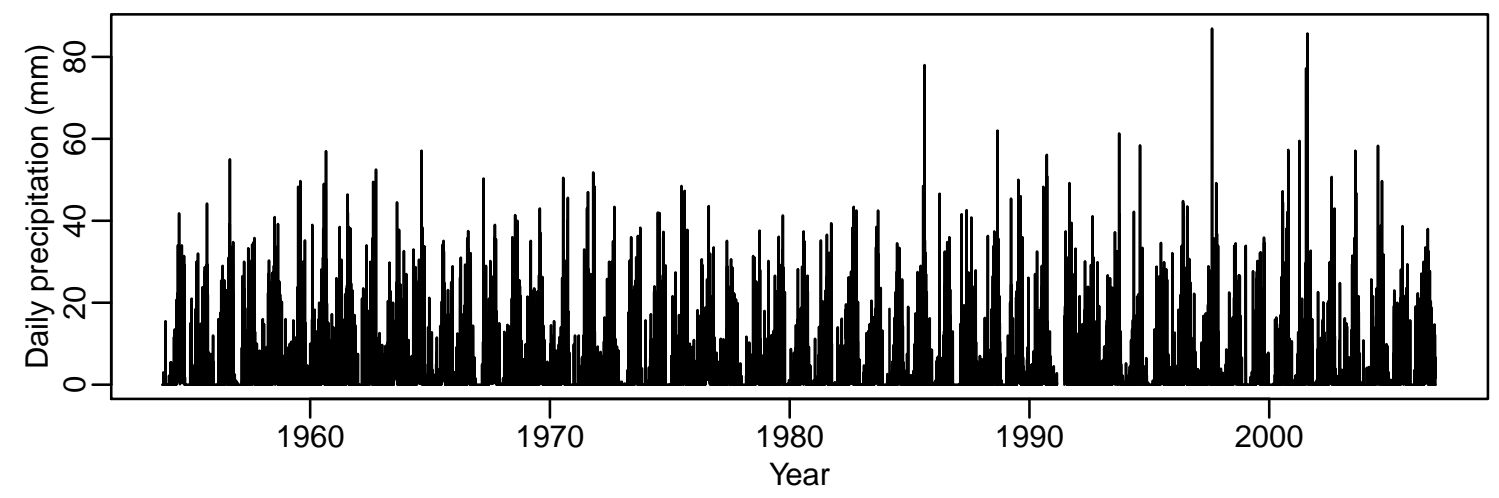

Fig. 1. Times series of daily precipitation at Debre Markos, Ethiopia.
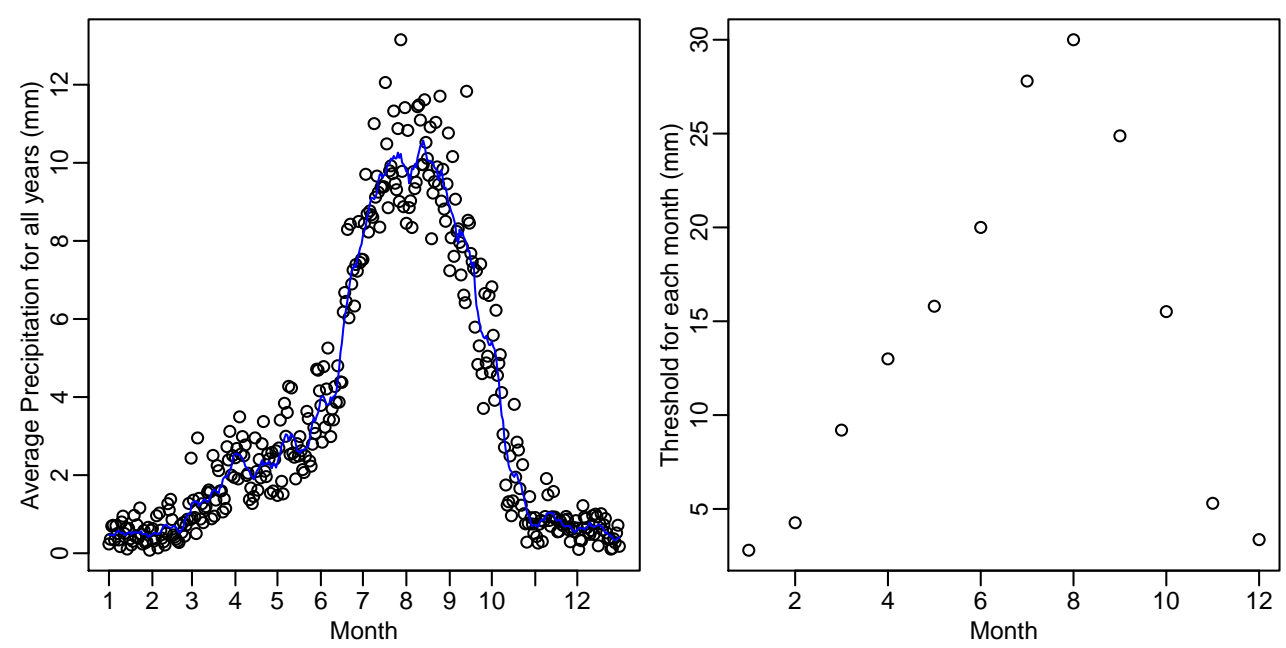

Fig. 2. Left: scatter plot of mean precipitation for each day overlaid with the 11-day moving average. Right: threshold chosen for each month.

than $5 \%$, which confirms that there is temporal dependence and hence the declustering is necessary.

Strong seasonality naturally exists in the data. As most areas in Ethiopia, there are three seasons in Debre Markos: main rainy season (June to September), dry season (October to January), and small rainy season (February to May), which are locally known as Kiremt, Bega, and Belg, respectively. Figure 2 (left panel) shows the mean precipitation for each day in a year, with the 11-day moving average overlaid. The plot is consistent with the three seasons. High precipitations are observed in summer months and low precipitations are observed in winter months. Our extreme value analysis needs to take the clustering and seasonality into account.

\section{Methods}

The basis of extreme value modeling is the GEV distribution, with distribution function

$F(z ; \mu, \sigma, \xi)=\left\{\begin{array}{l}\exp \left\{-\left[1+\xi\left(\frac{z-\mu}{\sigma}\right)\right]^{-1 / \xi}\right\}, \xi \neq 0, \quad 1+\xi\left(\frac{z-\mu}{\sigma}\right)>0, \\ \exp \left\{-\exp \left[-\frac{z-\mu}{\sigma}\right]\right\}, \xi=0\end{array}\right.$

where $\mu \in \mathbb{R}$ is a location parameter, $\sigma>0$ is a scale parameter, and $\xi \in \mathbb{R}$ is a shape parameter governing the tail behavior. The Gumbel family is the limiting case of $\xi \rightarrow 0$. The sub-families defined by $\xi>0$ and $\xi<0$ correspond to the Fréchet family and the Weibull family, respectively. The $m$-yr return level $z_{m}$, with the return period $1 / m$, is calculated from $F\left(z_{m}\right)=1-1 / m$. When the only available data is a sequence of annual maxima of daily precipitation, the maximum likelihood approach can be applied to make inferences about the unknown parameters. Usual regularity conditions of the maximum likelihood estimator are satisfied when $\xi>-0.5$ (Smith, 1985). 
With daily precipitation available, the POT approach and the point process approach are more attractive in that all exceedances over threshold, instead of just the annual maxima, contribute to the inference. Assuming that $X_{1}, \ldots, X_{n}$ are independent and identically distributed, Pickands (1971) showed that, for sufficiently large threshold $u$, the sequence of point processes $\left\{\left(i /(n+1), X_{i}\right): i=1, \ldots, n\right\}$ is approximated by a Poisson process on the region $(0,1) \times[u, \infty)$ with intensity function on $A=\left(t_{1}, t_{2}\right) \times[z, \infty)$ given by

$\Lambda(A)=n_{x}\left(t_{2}-t_{1}\right)\left[1+\xi\left(\frac{z-\mu}{\sigma}\right)\right]^{-1 / \xi}$,

where $n_{x}$ is the number of years of data to which the available $X_{i}$ correspond, ensuring that the parameters $(\mu, \sigma, \xi)$ are the same as those in the GEV approximation (Eq. 1) of annual maxima. The point process approach is adopted because the parameter estimates are not directly tied to the choice of threshold $u$ and the ideal threshold is determined by considering the smallest $u$ beyond which the parameter estimates stabilize.

Suppose that we observe $k$ exceedances of daily precipitation over threshold $u, x_{1}, \ldots, x_{k}$, from $n_{x}$ year's of data. The likelihood function is

$L\left(\mu, \sigma, \xi ; x_{1}, \ldots, x_{k}\right)=$
$\exp \left\{-n_{x}\left[1+\xi\left(\frac{u-\mu}{\sigma}\right)\right]^{-1 / \xi}\right\} \prod_{i=1}^{k} \sigma^{-1}\left[1+\xi\left(\frac{x_{i}-\mu}{\sigma}\right)\right]^{-1 / \xi-1}$.

The point process likelihood is based on all data greater than $u$, thus inferences are likely to be more accurate than estimates based on the classical GEV model which studies only block maxima. The likelihood also takes into account of missing data in that where there are missing data, $n_{x}$ will be the number of year's worth of observed data.

So far we have assumed that the data are independent and identically distributed, which is clearly violated in the daily series data. Before we can apply the likelihood function, we need to remove the clustering and seasonality from the observed data.

We use runs algorithm to filter the dependent observations to obtain a set of threshold excesses that are approximately independent (Smith and Weissman, 1994). For a given threshold, define clusters to be wherever there are consecutive exceedances of this threshold. In particular, two exceedances of the threshold that are separated apart by fewer than $r$ observations are deemed part of the same cluster. That is, only after a certain number, $r$, of observations fall below the threshold, the cluster is terminated. In practice, it is recommended to try different $r$ values for comparison (Smith, 1989; Mannshardt-Shamseldin et al., 2010).

To handle the seasonality, we adopt a simple and broadly applicable approach that allows all model parameters of the Poisson process to be seasonally dependent. Specifically, we allow each month to have its own GEV parameters as in Smith (1989).
Finally, how does one select the threshold $u$ ? Although the value of threshold can be arbitrary to some extent for initial analysis, too low a threshold is likely to violate the asymptotic basis of the model and too high a threshold will lead to too few exceedances for data analysis. An exploratory tool for choosing $u$ is the mean residual life plot (e.g., Coles, 2001, Ch. 4). When $u$ is sufficiently large, the expected residual life, $E(X-u \mid X>u)$, is a linear function of $u$. In a mean residual life plot, we plot the sample mean residual life against threshold $u$, and choose the smallest $u$ beyond which the mean residual life plot is approximately linear.

\section{Results}

The mean residual plots with $95 \%$ confidence intervals are drawn for each month with run length $r=1$ in Fig. 3. For all months, the figures are approximately linear when the threshold exceeds the sample 95\% percentile. Therefore, we take the $95 \%$ percentile as threshold for each month. This is different from the analysis of Smith (1989), where the same threshold was used for all months. The right panel of Fig. 2 shows the thresholds we choose for each month, which has similar pattern as the average precipitation plot in the left panel.

Each month is modeled separately, thus no specific form describing the seasonal variation is assumed. Let $\mu_{i j}, \sigma_{i j}$ and $\xi_{i j}$ denote the GEV parameters for month $j$ of year $i$. To detect the long-term trend for each month, we assume the form

$\mu_{i j}=\alpha_{j}+i \beta_{j}, \quad \sigma_{i j}=\sigma_{j}>0, \quad \xi_{i j}=\xi_{j}$,

where the location parameter $\mu_{i j}$ includes a linear trend in year with coefficient $\beta_{j}$. This form was also adopted to detect trend by Smith (1989) with ground-level ozone and by Cooley (2009) with annual maximum temperatures. The likelihood $L_{j}$ of month $j, j=1, \ldots, 12$, is maximized separately to estimate $\left(\alpha_{j}, \beta_{j}, \sigma_{j}, \xi_{j}\right)$.

It turns out that none of the $\beta_{j}$ parameters is significant at $5 \%$ level, indicating there is no strong evidence of longterm increasing trend over time. The models are re-fitted with all $\beta_{j}=0$. The sum of the maximized log likelihood is -3063.91 for the models in all 12 months, which is very close to that with $\beta_{j}$ 's in the model $(-3060.29)$. The parameter estimates with no trend are shown in Table 1. There is strong seasonal pattern for the location parameter $\mu$. The other parameters $\sigma$ and $\xi$, however, vary haphazardly. All $\xi$ 's are estimated greater than -0.5 , indicating that the estimators are regular and they have the usual asymptotic properties. The 10-yr return level for each specific month, calculated from GEV distribution, is also shown in the table.

The $95 \%$ confidence intervals for parameter estimates are calculated by profile likelihood (Coles, 2001, Ch. 2), which is shown in Fig. 4. Although the confidence interval of $\xi$ covers zero in all months, we do not reduce the model to the 

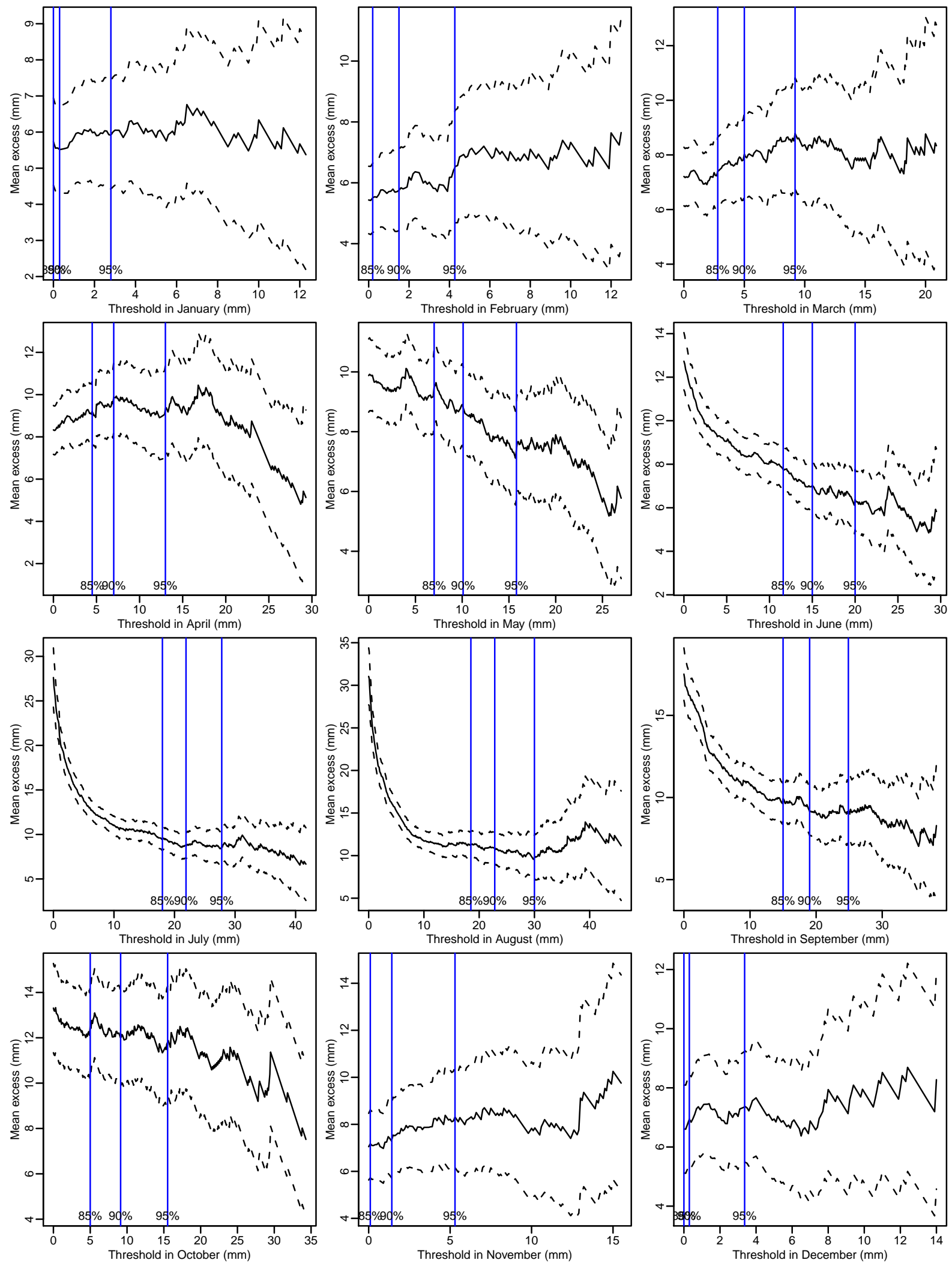

Fig. 3. Mean residual life (solid lines) with $95 \%$ confidence intervals (dashed lines) for all months with $r=1$. 

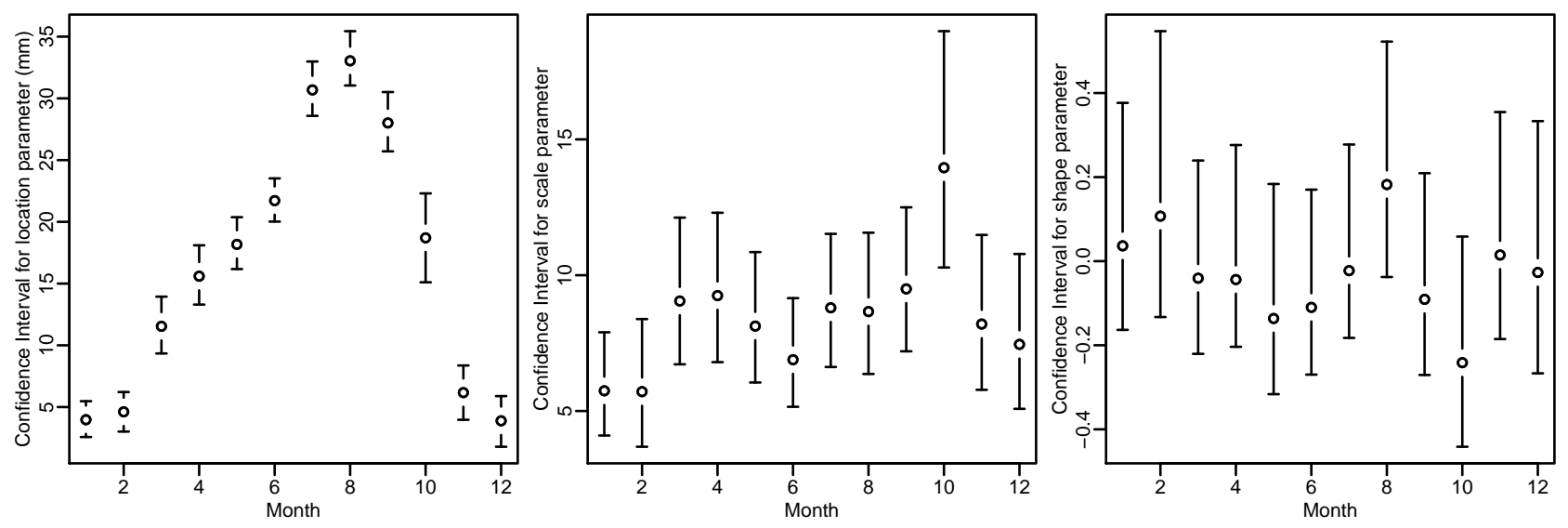

Fig. 4. 95\% confidence intervals for GEV parameters. Left: confidence intervals for $\mu$. Middle: confidence intervals for $\sigma$. Right: confidence intervals for $\xi$.

Table 1. Parameter estimates and standard errors for each month with no trend.

\begin{tabular}{ccrrrr}
\hline Month & $\begin{array}{c}\text { Number of } \\
\text { Exceedances }\end{array}$ & $\mu$ & $\xi$ & $\xi$ & $\begin{array}{c}\text { 10-yr return } \\
\text { level }\end{array}$ \\
\hline 1 & 65 & $3.97(0.74)$ & $5.74(0.96)$ & $0.04(0.14)$ & 17.45 \\
2 & 56 & $4.61(0.77)$ & $5.71(1.20)$ & $0.11(0.17)$ & 19.15 \\
3 & 67 & $11.54(1.17)$ & $9.05(1.35)$ & $-0.04(0.12)$ & 31.00 \\
4 & 68 & $15.60(1.20)$ & $9.25(1.38)$ & $-0.04(0.12)$ & 35.42 \\
5 & 69 & $18.18(1.05)$ & $8.13(1.21)$ & $-0.14(0.13)$ & 33.92 \\
6 & 67 & $21.72(0.88)$ & $6.89(1.01)$ & $-0.11(0.11)$ & 35.46 \\
7 & 72 & $30.68(1.12)$ & $8.80(1.23)$ & $-0.02(0.11)$ & 50.00 \\
8 & 75 & $33.04(1.12)$ & $8.66(1.34)$ & $0.18(0.14)$ & 57.14 \\
9 & 73 & $28.01(1.21)$ & $9.50(1.34)$ & $-0.09(0.12)$ & 47.34 \\
10 & 66 & $18.71(1.80)$ & $13.96(2.17)$ & $-0.24(0.13)$ & 42.94 \\
11 & 60 & $6.17(1.07)$ & $8.20(1.46)$ & $0.01(0.14)$ & 24.94 \\
12 & 57 & $3.89(0.99)$ & $7.45(1.44)$ & $-0.03(0.15)$ & 20.16 \\
\hline
\end{tabular}

Gumbel model with constraint $\xi=0$, because "a reduction to the Gumbel subfamily is always risky" (Coles and Pericchi, 2003, p. 416); the uncertainty in parameter $\xi$ would otherwise be inappropriately accounted for.

To check the sensitivity of results to the choice of threshold $u$ and run length $r$, return levels are compared under different choices. Since there is seasonality during the year, the calculation of the return level can be derived through the maxima for each month. Let $M_{1}, \ldots, M_{12}$ denote the maxima for each month. The $m$-yr return level $z_{m}$ will satisfy

$$
\begin{aligned}
1-\frac{1}{m}= & \\
& \operatorname{Pr}\left\{\max \left(M_{1}, \ldots, M_{12}\right) \leq z_{m}\right\} \\
= & \prod_{i=1}^{12} \exp \left\{-\left[1+\xi_{i}\left(\frac{z_{m}-\mu_{i}}{\sigma_{i}}\right)\right]^{-1 / \xi_{i}}\right\} .
\end{aligned}
$$

The confidence interval for return level can be obtained by simulation. We simulate the model parameters first from the the multivariate normal approximation of the estimator. For each set of generated parameters, a realization of the return level is obtained by solving Eq. (5). A large number $(N=5000)$ of realizations is used to approximate the confidence intervals.

Table 2 summarizes the parameter estimates and $95 \%$ confidence intervals for 10-yr, 50-yr and 100-yr return levels for different combinations of $(u, r)$. It appears that the inference is quite robust on the choice of $r$ for all return levels. The inference on the 10-yr return level is robust to the choice of $u$, but the 50-yr and 100-yr return levels are less so, which is most evident from the upper bound of the $95 \%$ confidence interval. The change in confidence intervals is not completely surprising because the sample size of exceedances decreases as $u$ increases. With the confidence intervals in consideration, the changes in the point estimate of return levels appear reasonably robust. 
Table 2. Estimated return levels and their $95 \%$ confidence intervals under different choices for threshold $u$ and run length $r$.

\begin{tabular}{lcccc}
\hline$u$ & $r$ & 10-yr return level & 50-yr return level & 100-yr return level \\
\hline$Q_{85 \%}$ & 1 & $69.0(64.6,78.4)$ & $90.0(82.3,117.6)$ & $100.5(91.3,147.8)$ \\
$Q_{85 \%}$ & 2 & $69.0(64.5,78.5)$ & $89.8(81.8,118.5)$ & $100.1(89.6,154.0)$ \\
$Q_{90 \%}$ & 1 & $68.6(63.5,79.0)$ & $91.4(82.0,122.8)$ & $103.4(90.8,154.6)$ \\
$Q_{90 \%}$ & 2 & $68.5(63.3,78.7)$ & $90.6(80.9,119.6)$ & $102.0(88.7,146.6)$ \\
$Q_{95 \%}$ & 1 & $68.4(61.7,80.8)$ & $97.2(80.5,142.2)$ & $113.4(89.5,186.5)$ \\
$Q_{95 \%}$ & 2 & $68.4(61.8,80.5)$ & $96.8(80.0,141.3)$ & $112.7(89.8,184.0)$ \\
$Q_{97 \%}$ & 1 & $68.1(61.3,81.2)$ & $99.3(79.6,155.4)$ & $118.0(90.1,223.4)$ \\
$Q_{97 \%}$ & 2 & $67.9(61.1,80.1)$ & $98.3(78.5,153.4)$ & $116.4(88.0,208.9)$ \\
\hline
\end{tabular}

Among all those threshold sets, the only significant $\beta_{j}$ 's were found when $u=Q_{90 \%}$ and $r=1$, with standardized beta values -2.07 and -2.15 for February and July, respectively. We conclude that there is no increasing long-term trend for any month.

As a model diagnosis, we performed goodness-of-fit test for the GEV distribution with the annual maximum daily precipitation data in each of the 12 months over $53 \mathrm{yr}$. There were 10, 10, and 13 zeros in January, February, and December, respectively. These zeros were removed to run the goodness-of-fit test as, otherwise, a distribution with point mass at zero would be needed and any continuous distribution would fail to capture this. For the POT approach, these zeroes would not affect the result as they do not affect the selection of the threshold. The $p$-values of the KolmogorovSmirnov test statistic are, respectively, 0.405, 0.220, 0.197, $0.127,0.674,0.621,0.562,0.560,0.313,0.465,0.494$, and 0.372 from January to December, suggesting no lack of fit from the GEV distribution. The $p$-values of the AndersonDarling test give similar results.

Finally, we present the estimated return level plots for the model with no trend in Fig. 5. The $95 \%$ confidence intervals were obtained again by a large number $(N=5000)$ of Monte Carlo simulation that accounts for the uncertainty in parameter estimate. The 100-yr return level was estimated as 96.4, with a $95 \%$ confidence interval $(78.7,161.0)$.

\section{Conclusions}

With the extreme value theory, we presented a case study with the daily precipitation series at Debre Markos, Ethiopia. No evidence was found to support long-term increasing trend in extreme precipitation at this location. This means, for instance, that the 100-yr return level has not increased significantly during the period of 1953-2006. We have performed the same analysis with daily records separately at two other sites, Bahir Dar and Gondar, in the Blue Nile River basin on the Northwestern Highland in Ethiopia. No significant trend was found at either sites.

In practice, for a given data set, many parametric families may fit the data well and pass the goodness-of-fit test.

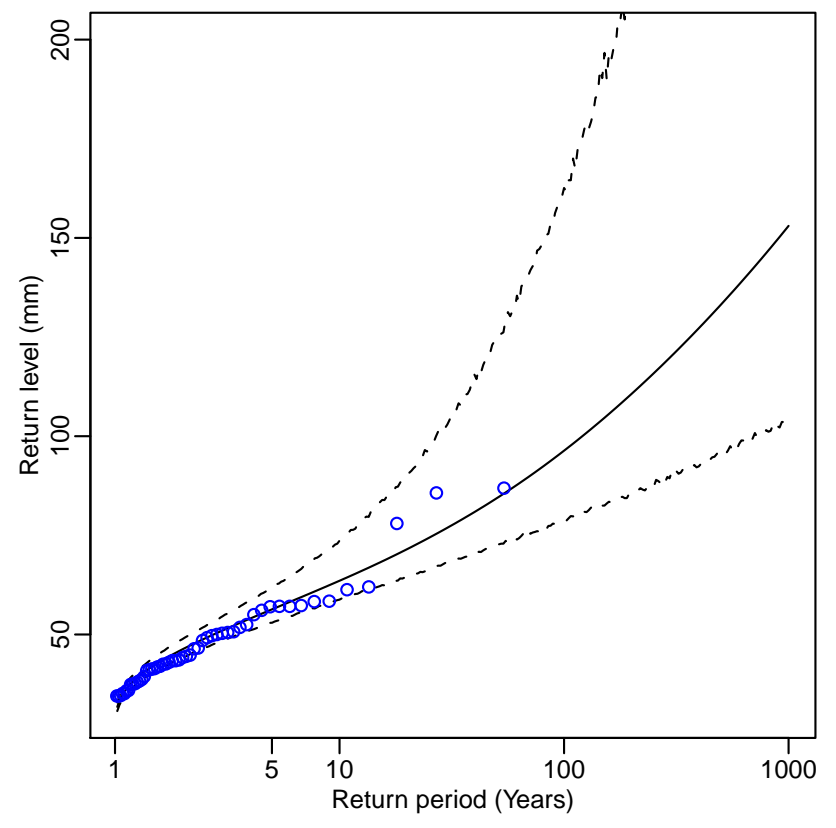

Fig. 5. Return levels (solid line) with $95 \%$ confidence intervals (dashed lines) obtained from 5000 Monte Carlo simulation. The circles are the empirical estimates based on the observed 53-yr's data.

One can always maximize the likelihood under the assumption that the data come from an assumed family, which is likely a misspecification of the real distribution (White, 1982). As the true distribution is unknown, the fitted distribution for any assumed parametric family from the maximum likelihood approach is the one in this assumed family that minimizes the Kullback-Leibler divergence (e.g., Kullback, 1987). Models from different families are in general not nested, and to perform model selection, one can use Vuong's test (Vuong, 1989), which chooses the model with the least Kullback-Leibler divergence. Nevertheless, distinguishing two nonnested models with statistical significance requires a large amount of data when competing models offer similar capabilities in capturing the observed data frequencies. With only 53 observations, other distributions such as generalized 
Pareto, fatigue life, and lognormal may fit the data as well as GEV. These distributions, however, can differ very much in tails, which is what we want to study through extreme value analysis. For this reason, a GEV model may be preferred as it is by definition the limit distribution of sample maximums.

Our current extreme value analysis deals one site at a time. It cannot address important questions that involve events jointly defined across multiple sites; for instance, what is the probability that the 100-yr return levels of three sites in the vicinity of a city occur in the same year? Estimating the probability of extremal events at a network of locations with spatial dependence appropriately accounted is a much more challenging problem. Spatial extremes is a new and rapidly developing field (e.g., Cooley et al., 2007; Padoan et al., 2010). Further extreme analysis in a spatial context for Ethiopia, with data from a network of sites, is worth investigating.

Acknowledgements. H. Shang and J. Yan were partially supported by a grant from the University of Connecticut Research Foundation. M. Gebremichael was partially supported by NASA NIP Grant No. NNX08AR31G and NASA Grant No. NNX10AG77G to the University of Connecticut.

Edited by: A. Melesse

\section{References}

Beirlant, J., Goegebeur, Y., Segers, J., and Teugels, J.: Statistics of Extremes: Theory and Applications, John Wiley \& Sons Inc, 2004.

Cheung, W. H., Senay, G. B., and Singh, A.: Trends and Spatial Distribution of Annual and Seasonal Rainfall in Ethiopia, Int. J. Climatol., 28, 1723-1734, 2008.

Coles, S.: An Introduction to Statistical Modeling of Extreme Values, Springer-Verlag Inc, 2001.

Coles, S. and Pericchi, L.: Anticipating Catastrophes through Extreme Value Modelling, J. Roy. Stat. Soc. C-App., 52, 405-416, 2003.

Cooley, D.: Extreme Value Analyis and the Study of Climate Change: A Commentary on Wigley 1988, Clim. Change, 97, 7783, 2009.

Cooley, D., Nychka, D., and Naveau, P.: Bayesian Spatial Modeling of Extreme Precipitation Return Levels, J. Am. Stat. Assoc., 102, 824-840, 2007.

Durman, C. F., Gregory, J. M., Hassell, D. C., Jones, R. G., and Murphy, J. M.: A Comparison of Extreme European Daily Precipitation Simulated by a Global and a Regional Climate Model for Present and Future Climates, Q. J. Roy. Meteor. Soc., 127, 1005-1015, 2001.

Fisher, R. A. and Tippett, L. H. C.: Limiting Forms of the Frequency Distributions of the Largest or Smallest Member of a Sample, P. Camb. Philos. Soc., 24, 180-190, 1928.
Frei, C. and Schär, C.: Detection Probability of Trends in Rare Events: Theory and Application to Heavy Precipitation in the Alpine Region, J. Climate, 14, 1568-1584, 2001.

Goswami, B. N., Venugopal, V., Sengupta, D., Madhusoodanan, M. S., and Xavier, P. K.: Increasing Trend of Extreme Rain Events over India in a Warming Environment, Science, 314, 1442-1445, 2006.

Huerta, G. and Sansó, B.: Time-varying Models for Extreme Values, Environ. Ecol. Stat., 14, 285-299, 2007.

Karagiannidis, A., Karacostas, T., Maheras, P., and Makrogiannis, T.: Trends and Seasonality of Extreme Precipitation Characteristics related to Mid-latitude Cyclones in Europe, Adv. Geosci., 20, 39-43, doi:10.5194/adgeo-20-39-2009, 2009.

Kharin, V. V. and Zwiers, F. W.: Estimating Extremes in Transient Climate Change Simulations, J. Climate, 18, 1156-1173, 2005.

Kullback, S.: The Kullback-Leibler Distance [Letter], The American Statistician, 41, 340-341, 1987.

Kunkel, K. E.: North American Trends in Extreme Precipitation, Nat. Hazards, 29, 291-305, 2003.

Kunkel, K. E., Andsager, K., and Easterling, D. R.: Long-Term Trends in Extreme Precipitation Events over the Conterminous United States and Canada, J. Climate, 12, 2515-2527, 1999.

Mannshardt-Shamseldin, E. C., Smith, R. L., Sain, S. R., Mearns, L. O., and Cooley, D.: Downscaling Extremes: A Comparison of Extreme Value Distributions in Point-source and Gridded Precipitation Data, The Annals of Applied Statistics, 4, 484-502, 2010.

Padoan, S. A., Ribatet, M., and Sisson, S. A.: Likelihood-based Inference for Max-stable Processes, J. Am. Stat. Assoc., 105, 263277, 2010.

Pickands, J.: The Two-dimensional Poisson Process and Extremal Processes, J. Appl. Probab., 8, 745-756, 1971.

Seleshi, Y. and Camberlin, P.: Recent Changes in Dry Spell and Extreme Rainfall Events in Ethiopia, Theor. Appl. Climatol., 83, 181-191, 2006.

Seleshi, Y. and Zanke, U.: Recent Changes in Rainfall and Rainy Days in Ethiopia, Int. J. Climatol., 24, 973-983, 2004.

Smith, R. L.: Maximum Likelihood Estimation in a Class of Nonregular Cases, Biometrika, 72, 67-90, 1985.

Smith, R. L.: Extreme Value Analysis of Environmental Time Series: An Application to Trend Detection in Ground-level Ozone (C/R: P378-393), Stat. Sci., 4, 367-377, 1989.

Smith, R. L. and Weissman, I.: Estimating the Extremal Index, J. Roy. Stat. Soc. B, 56, 515-528, 1994.

Vuong, Q. H.: Likelihood Ratio Tests for Model Selection and Nonnested Hypotheses (STMA V31 0456), Econometrica, 57, 307333, 1989.

White, H.: Maximum Likelihood Estimation of Misspecified Models, Econometrica, 50, 1-26, 1982.

Zhang, X., Zwiers, F. W., and Li, G.: Monte Carlo Experiments on the Detection of Trends in Extreme Values, J. Climate, 17, 1945-1952, 2004. 